\title{
Minimum-component baselines: Fourier analysis for continuum assessment
}

\author{
J.V. Wall \\ Royal Greenwich Observatory, Madingley Road, Cambridge CB30EZ, UK
}

Received November 22, 1994; accepted July 22, 1996

\begin{abstract}
This describes a technique for fitting baselines or continua to scans or spectra with a large proportion occupied by signal in the form of sources or emission/absorption lines. It is essentially a high-pass filtering technique; the very lowest Fourier frequencies are "filtered out" by using them to construct a model of the continuum. The technique is interactive, and subjective in that some decision is required by the analyst; but it is very stable despite this. Errors are analyzed and some examples are given to demonstrate the versatility of the technique. The route to generating an objective (hands-off) technique is discussed.
\end{abstract}

Key words: methods: data analysis; numerical techniques: image processing

\section{Introduction: The problem}

In astronomy as in many physical sciences there is frequent need to measure signal from a data series. In measuring a specific attribute of this signal such as redshift, the power of Fourier analysis has long been recognized (e.g. Sargent et al. 1977; Tonry \& Davis 1979). It is the purpose here to draw attention to the power of Fourier analysis in the less well-defined case of attempting general signal measurements from a data series.

This problem of accurate measurement of signal parameters inevitably comes down to assessment of "zerolevel" or continuum height. Many techniques are invoked - from eye-ball sketching, through least-squares fits of polynomials of low orders, heavy-smoothing, and splinefitting. The difficulty is inevitably the signal. Those parts of the scan with signal must be removed from consideration in order to place the continuum; and with irregular and a priori unknown spacing of the signal, development of a formal technique becomes prejudicial or perhaps impossible. Moreover, smoothing techniques and polynomial fits make initial assumptions which the data may not justify. For some types of signal such as emission or absorption lines with extreme breadth of wings, the behaviour of the continuum in the regions masked by signal is critical in measurement of that signal.

There are formal tools to apply. For example Bayesian spectral analysis (e.g. Sivia \& Carlile 1992) is appropriate when some specific prior knowledge such as line-width is available. However the analysis must be repeated for each different prior-knowledge set and for each different question posed of the data. The situation frequently arising in spectral analysis is one in which the prior knowledge is the somewhat unquantifiable recognition of which parts of the spectrum are signal-free, while very general parameter sets (e.g. line-shape, line-width, line-flux, equivalent width, centroid position) may be required from the measurements.

A simple harmonic-analysis technique is described here which provides a good basis for such analysis, and which can be optimized in cases with some a priori knowledge of signal form. It gives results which are stable from observer to observer.

There will always remain instances in which baseline assessment is impossible, even additional resolution being unable to rescue a continuum such as Ly $\alpha$ forests in QSO spectra, some regions of the solar spectrum, and the spectra of stars of types much later than solar. These represent uni-directional confusion of signal, absorbed or negative signal in these cases, with no possibility of true continuum appearing on both sides of a signal-dominated area to give the analyst a chance. Such cases must be dealt with by techniques beyond the scope of this note, techniques involving a priori knowledge or models of instrument performance and line patterns. However, cases of pure confusion of signal in which the probabilities of positive and negative deflections are equal, do lend themselves to baseline assessment of the type described here (Wall et al. 1982).

The paper is concerned with data sets of the type represented by the optical spectra in the upper panels of Fig. 1 and Fig. 2. In the first case, the spectral appearance is dominated by substantial emission lines covering more than $30 \%$ of the length of the spectrum. In the second case, the continuum slope is severe and the 
spectrum is dominated by broad and deep absorption lines.
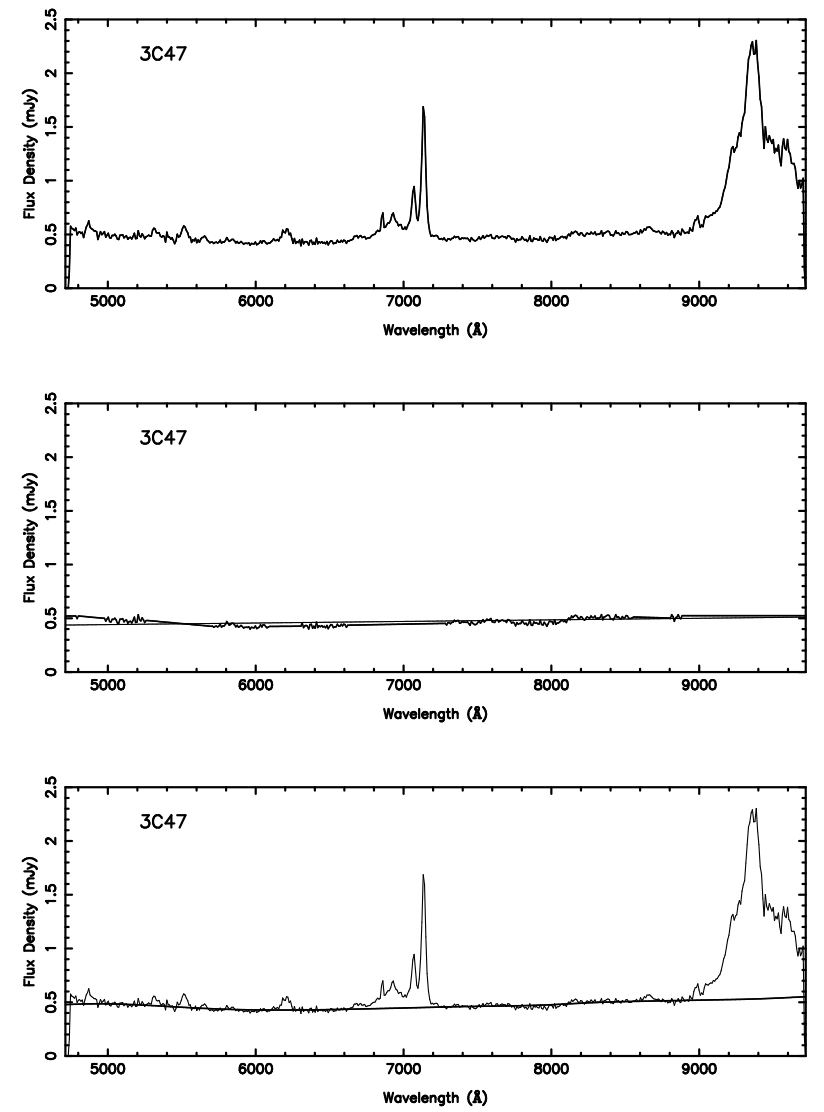

Fig. 1. A spectrum of 3C 47 obtained by Laing et al. (1994) with the Faint Object Spectrograph of the William Herschel Telescope, La Palma. The redshift is 0.345 ; broad lines of the hydrogen Balmer series can be seen, together with narrow lines of [OIII]. The upper panel shows the flux-calibrated spectrum, while the middle panel shows the "patched" spectrum, the scan with portions of apparent signal patched out, together with a straight-line least-squares fit. The lower panel shows the continuum obtained from using the low-frequency Fourier components of this patched spectrum, superposed on the original data

\section{A solution}

A solution lies in taking the one-dimensional scan to pieces in a Fourier analysis. Fourier theory (e.g. Bracewell 1965) indicates that any continuous function may be represented as the sum of sines and cosines, i.e.

$f(t)=\int_{-\infty}^{+\infty} F(\omega) \exp ^{-i \omega t} \mathrm{~d} \omega$

where the function $F$ representing the phased amplitudes of the sinusoidal components of $f$ is known as the Fourier
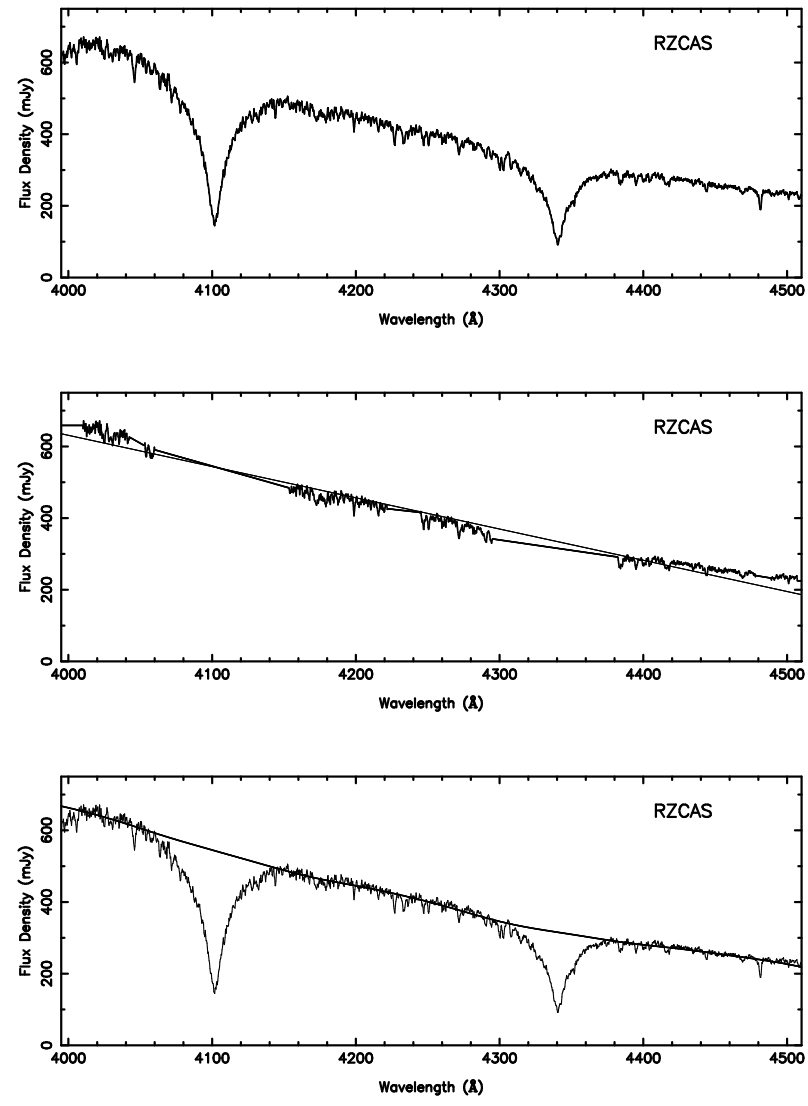

Fig. 2. A spectrum of RZ Cas (Maxted et al. 1994). The panels are as described in the caption for Fig. 1

Transform (FT). If the function is sampled $N$ times at uniform intervals $\Delta t$ in the spatial (observed) frame, the total length in the $t$ - direction is $L=\Delta t \times(N-1)$, and the result is the continuous function multiplied by the "comb" function, producing a function $f^{\prime}(t)$ which (with the interval in spatial frequency as $\Delta \nu=\frac{2 \pi}{\Delta t}$ ) may be represented (e.g. Gaskill 1978) either as a sum of sines and cosines

$f^{\prime}(t)=A_{n} \Sigma \sin (n \Delta \nu)+B_{n} \Sigma \cos (n \Delta \nu) ;$

or as a cosine series

$f^{\prime}(t)=A_{n}^{\prime} \Sigma \cos \left(n \Delta \nu+\Phi_{n}^{\prime}\right)$

where amplitudes $A_{n}^{\prime}$ and phases $\phi_{n}^{\prime}$ are given by

$A^{\prime}{ }_{n}=\sqrt{A^{2}{ }_{n}+B^{2}{ }_{n}}, \phi_{n}^{\prime}=\arctan \left(\frac{A_{n}}{B_{n}}\right)$.

In the latter formulation, obtaining the FT produces by virtue of the $2 \pi$ cyclic nature of sine and cosine - a "Fourier-Transform plane" for $f^{\prime}(t)$ which shows the amplitudes mirror-imaged about zero frequency, with a sampling in spatial frequency at intervals of $\frac{2 \pi}{\Delta t(N-1)}$ and a repetition of the pattern at intervals of $\frac{2 \pi}{\Delta t}$. 
There are three criteria for successful discretesampling.

1. The Nyquist criterion or Nyquist limit guarantees that there is no information at spatial frequencies above $\frac{\pi}{\Delta t}$. (Consider the silly case of a signal which is a spatial sine wave of wavelength $2 \Delta t$ : sampling at intervals of $\Delta t$ finds points of identical amplitude and thus does not carry information on amplitude or phase of this spatial frequency.) Thus the sampling interval $\Delta t$ sets the highest spatial frequency $\frac{2 \pi}{\Delta t}$ which can be present; if higher frequencies are present in the data, this sampling rate loses them.

At the same time, the sampling theorem (Whittaker 1915; Shannon 1949) indicates that any bandwidthlimited function can be specified exactly by regularlysampled values provided that the sample interval does not exceed a critical length (which corresponds approximately to half the FWHM resolution), i.e. if the instrumental half-width is $B, f^{\prime}(t) \rightarrow f(t)$ if $\Delta t \leq \frac{B}{2}$.

2. To avoid any ambiguity - aliasing - in the reconstruction of the scan from its FT, the sampling interval must be small enough for the amplitude coefficients of components at frequencies as high as $\frac{\pi}{\Delta t}$ to be effectively zero. If $A_{n}^{\prime} \geq 0$ for components of frequency this high, the positive high-frequency tail of the repeating $A^{\prime}(\nu)$ tangles up with the negative tail of the symmetric function repeating about $\nu=\frac{2 \pi}{\Delta t}$ to produce an indeterminate transform.

3. Any physical system is indeed band-pass limited (although noise added by the subsequent detector is not necessarily so), and is a low-pass filter; it is in the accurate assessment of the lowest spatial frequencies, the slowly-varying continuum, where our interest lies. The lowest frequencies which harmonic analysis can delineate are at $\frac{2 \pi}{N \Delta t}$. Such low-frequency spatial components may be real as in the case of a stellar spectrum, or may be instrumental in origin as for sky scans with a single-beam radio telescope. In either case, to have any chance of distinguishing these from those of the signal, the scan length must exceed the width of single resolved features by a factor preferably $\geq 10$.

In practice most data satisfy these properties. By design, sampling is frequent enough to maintain resolution, to obtain spatial frequencies beyond those present in signal, and to avoid aliasing. By design we take spectra over ranges substantially greater than the width of the features. Consider for instance the FT of the spectra in the upper panels of Figs. 1 and 2, whose $A_{n}^{\prime}$ are shown in upper panels of Fig. 3 and Fig. 4. Only half the interval of the FT is shown in these plots, up to $\frac{\pi}{2 \Delta t}$; the amplitude of the components has drained away very satisfactorily. It is therefore appropriate to consider what can be done to such scans via harmonic analysis, filtering in the Fourier (frequency) plane, and reconstruction.
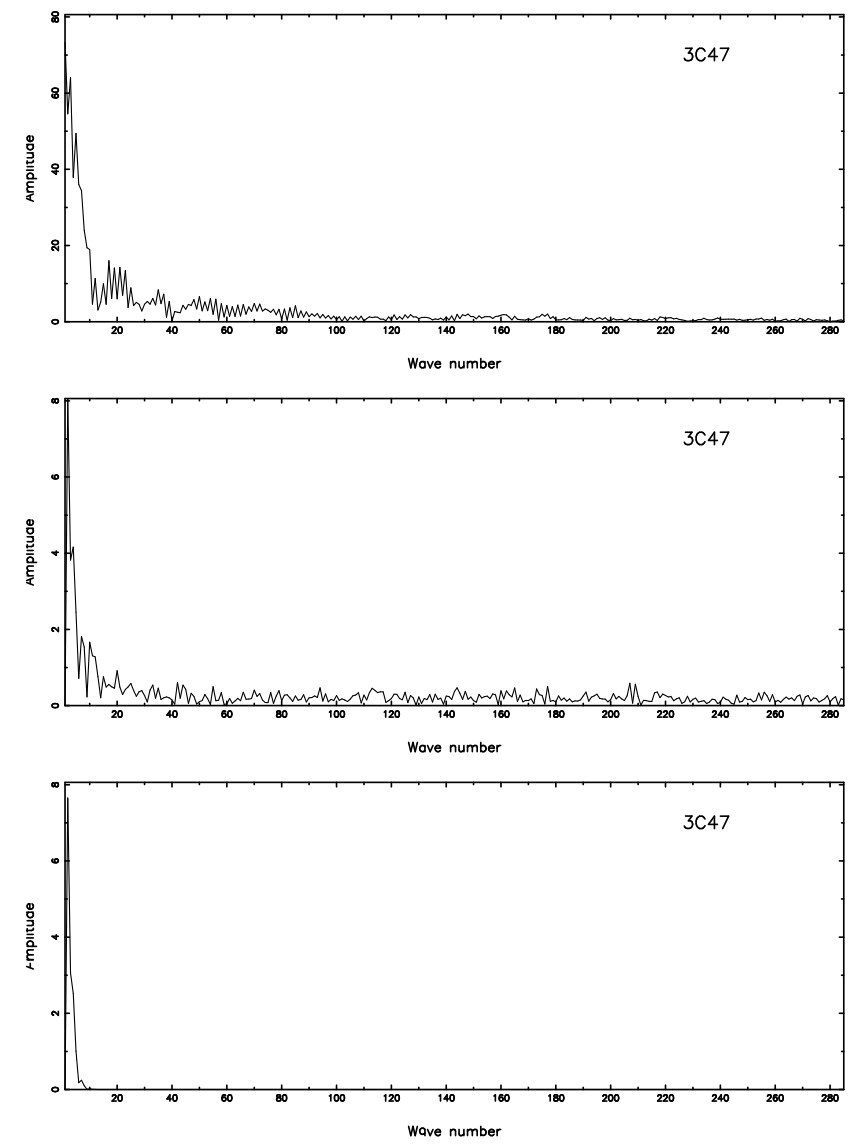

Fig. 3. Upper panel: The amplitudes of the Fourier components of the spectrum in Fig. 1, top. Central panel: The amplitudes of the Fourier components of the patched spectrum (with the least-squares approximation subtracted) of Fig. 1, middle. Note the change in scale, due to the removal of the massive low-frequency signal present in the lines. Lower panel: The same amplitude plane after application of a half-Gaussian taper of scale $\sigma_{\mathrm{f}}=3.0$ channels. This is the set of amplitudes used in the reverse FT which produces the baseline shown in Fig. 1, lower panel

To fit a continuum or remove a baseline, one could in principle consider an optimum filter for removing the prevalent low-frequency components. The difficulty is the well-known property for the FT of a Gaussian to be a Gaussian in the Fourier plane; even unresolved lines thus have very low frequency components. The result is that the harmonic components which govern the baseline are also required for the signal. Indeed simply building a baseline by reverse-transforming the few lowest-frequency components from the transform of the spectrum will produce baselines with gross distortion in regions of signal due to the low-frequency signal components.

However, it is straightforward to "patch out" regions of signal, as shown in the central panels of Figs. 1 and 2 . A simple linear interpolation across suspect or manifestly "bad" (line) regions is easily selected and for the purpose 

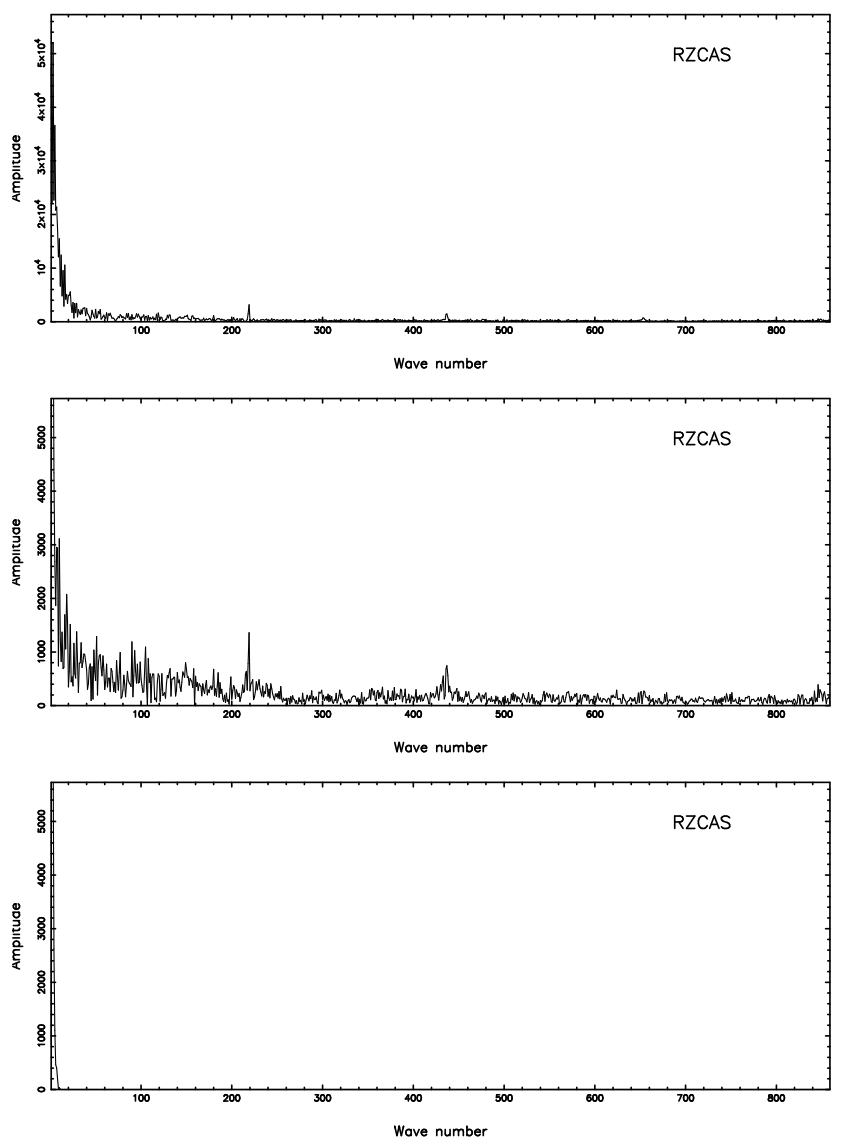

Fig. 4. The FT amplitudes of the complete scan (upper panel), the patched scan (central panel), and the filtered result from which the baseline is constructed (lower panel), as described in the caption for Fig. 3

interactive graphics can be used. Such a process is remarkably stable against the choice of the extent of the patches, except for extreme curvature of the baseline.

The next step is to take the FT of this "patched" array, and then to filter the low-frequency components from this. A formal method can be used, such as the filtering described by Martin (1959); but a simple and effective approach is to apply a savage half-Gaussian taper to the amplitude plane. (Any filter applied needs a taper to prevent ringing effects.) Finally the continuum is reconstructed with the inverse transform of the patched/filtered data.

The FT (amplitude) of the patched arrays are shown in the central panels of Figs. 3 and 4. The heavy halfGaussian filter applied in this instance has a $\sigma=3.0$ wave-numbers. The lower panels of Figs. 3 and 4 show the savagery of the filtering which, when the inverse transform is applied, yields the highly satisfactory continua in the lower panels of Figs. 1 and 2 .

The cyclic nature of the FT technique always means that the ends of the scan must match, so that an overall slope must be removed at the outset. A simple least- squares fitting technique, or very heavy smoothing to estimate a zero-order approximation to the continuum, can be used for this purpose, as shown in the central panels of Figs. 1 and 2.

To summarize, the baseline-finding technique advocated here consists of

1. patching: forming a "baseline array" from the original data-series by patching across regions of the scan where signal is evident;

2. end-matching: subtracting from this baseline array a first approximation to the patched scan, obtained with a linear fit, a very low-order polynomial or a heavysmoothing estimate;

3. Fourier Transforming the resultant baseline array;

4. Removal of the high frequencies by applying a heavytapered multiplicative filter to taper off the higherfrequency Fourier amplitudes;

5. Reverse-transforming using these minimum remaining components; and

6. Gradient-restoration, by adding back in the first approximation (step 2) to the baseline.

\section{Discussion}

\subsection{Criticism}

In some instances the cyclic nature of the FT + inversion process may render it unsuitable. The most intractable cases are those for which something happens at the scan ends which is different in physical process from that governing the general run of the scan. It may be that an undesirable amount of high-frequency component is needed in the filter to fit the extremeties, leaving the center of the baseline fluctuating too violently. Judicious pruning of scan-ends (which may contain suspect data anyway) may solve the problem.

But what about buried lines or faint broad lines? Or just plain faint lines which have not been patched? It is fair criticism that such line amplitudes will be reduced because the unseen signal was not "patched out", and the filter process has indeed removed some of the lowfrequency components which would have contributed to their amplitudes. However, no objective technique is going to correct for unseen features; and if features are strong enough to recognize or sense, they are strong enough to patch. Concern about the signal reduction which results in not patching very weak lines, signal which is statistically known, can be assessed by Monte-Carlo analysis (e.g. Wall et al. 1982).

Techniques of polynomial or spline fits, or of heavy smoothing, are generally less objective than Fourier analysis. They do not avoid the signal-reduction difficulty, and in each of these cases, some form of "patching" and treatment of the scan ends is going to be required. 

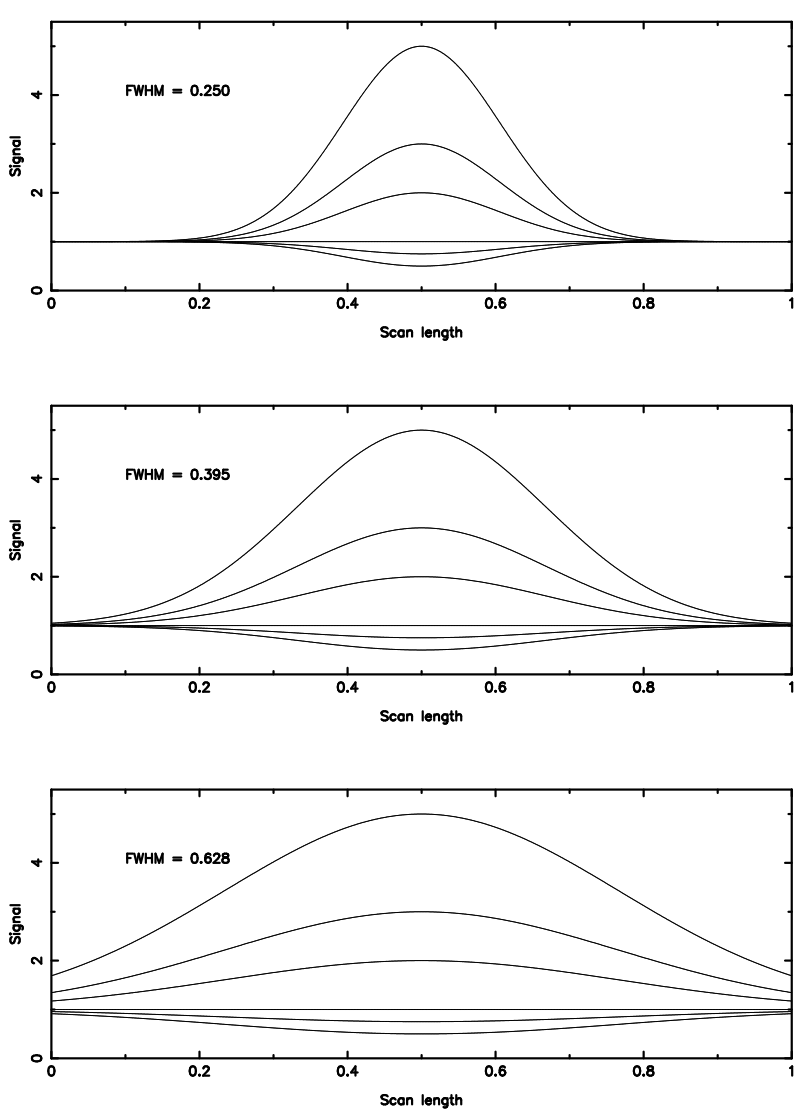

Fig. 5. Part of the set of distorted continua on which signals are placed to carry out the error analysis. Each continuum of unit length consists of a Gaussian of height $b$ added at the mid-point of a base of unity. The 5 values of $b$ are $4.0,2.0$, $1.0,-.25$ and -.50 . The calculations were carried out with 6 families of these baselines, the Gaussians having half-widths of $0.250,0.395,0.628,0.995,1.577$ and 2.500 scan-lengths. The first three of these are shown above

\subsection{Error analysis}

It is difficult to make a comparative error analysis; the numerous and ill-disciplined ways in which baselines or continua are normally derived do not provide standard models against which comparison can be made. One of the advantages of the technique described above is to enable a formal estimate of the error in signal and/or equivalent width which results from continuum assessment.

The minimum-component technique results in perfect fits and perfect flux measurement (except for noise) if the baseline is linear. It is only when signal sits on bumps or in hollows that error results. To carry out an analysis of such errors, a simple model of a continuum and a signal based on Gaussians was adopted. The geometry, shown in Fig. 5 and Fig. 6, consists of Gaussian signals of $\sigma_{\mathrm{s}}$ sitting at the maxima or minima of continua built of a unit dc level plus a centered Gaussian of height $b$ and given FWHM.
The data stream is of unit length and the Gaussian signal is of height $h(b+1)$, i.e. $h$ is the ratio of the signal height to the centrepoint of the continuum.
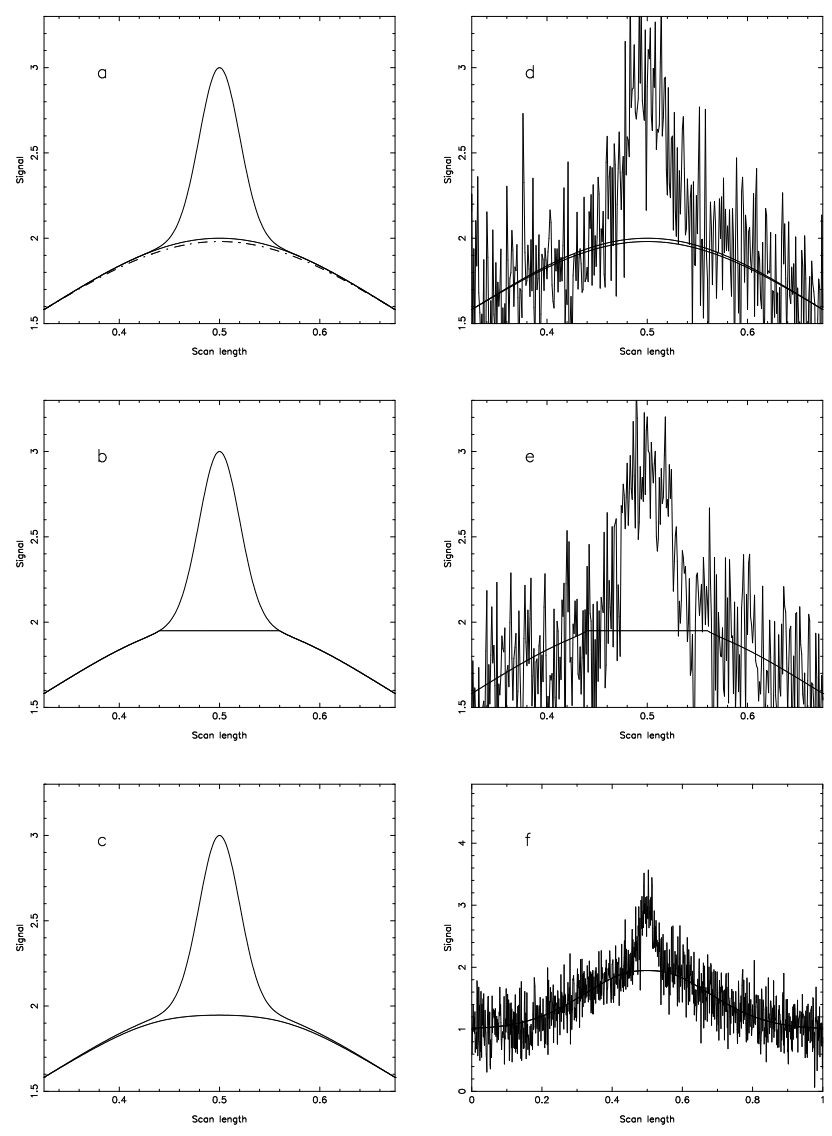

Fig. 6. Panel a) shows a Gaussian signal of $\sigma_{\mathrm{s}}=0.01$ sitting at the maximum of the member of the distorted-continuum family of Fig. 5 with $b=1.0, \mathrm{FWHM}=0.395$. The true baseline is the solid curve and the minimum-component baseline selected to give a $1 \%$ error is the dash-dot curve. The signal amplitude $h=0.5 \times(b+1)$. Panel $\mathbf{b})$ shows the total curve with a patch at $\pm 3 \sigma_{\mathrm{s}}$, while panel $\mathbf{c}$ ) is the resultant baseline after filtering the patched curve of $\mathbf{b}$ ) by limiting the Fourier components in the reconstruction of the patched approximation as described. Panels d) and e) are panels a) and b) with noise of $\mathrm{rms}=0.15 h$ added for verisimilitude, while panel $\mathbf{f}$ ) shows the entire scan length with the minimum-component continuum. In this example the error in equivalent width with the minimum-component baseline is $13 \%$; if $\sigma_{\mathrm{s}}$ is doubled at 0.02 , this error rises to $50 \%$

In this (or any) model of the continuum the sources of error in signal measurement are the following.

1. Badly-fitted continuum. If the continuum contains distortions whose scale-length approaches that of the signal, the problem of signal measurement becomes hopeless. In the case of astrophysical assessment, broad continuum features of this type may be due to a) poorly 
corrected instrumental response, or b) underlying signal features which are intrinsically broad, such as the blue bump in QSO spectra, the iron-line complexes in QSO spectra, or very broad line components. In these instances it is essential to decide what is to be measured and to design the baseline accordingly.

In the current methodology, it is possible that the baseline may be badly fit by not allowing enough Fourier components to take part in the assembly of the filtered baseline - we are trying to use the minimum number of components in order to produce the best approximation and to avoid including any noise or signal. The difficulty as illustrated in Fig. 6 may be that in the presence of noise, this badness of the fit goes unrecognized.

2. Deviation due to the patch. In the worst-case situation shown in Fig. 6, patching across the region on which the signal sits is going to exacerbate the poorness of fit of the baseline. The subsequent filtering which smooths the patch merely serves to drop the baseline yet futher away from the true level. The larger the patch in comparison with the scale of the baseline distortion, the larger this imposed deviation. Moreover, the more false signal from the "peak" of the continuum will be included as signal.

3. The summing of flux. The region over which the flux is summed is vital and must be constrained. Too large a region drives the baseline error to dominate the flux error totally. (The problem is well recognized in stellar photometry, in which it is customary to measure out to between 80 and 90 percent of the projected light i.e. down the Gaussian to $\sim 2 \sigma$ and to do so for standard stars and programme stars. But this is only applicable if the profiles or light distribution are identical for both programme and standard stars).

Note that if equivalent width is calculated, it exacerbates the errors, in the sense that the flux error due to the baseline error increases the measured equivalent width over the true, while the baseline error itself decreases the baseline and thus also increases the measured equivalent width over the true.

Even with the simplistic model of Fig. 5 and Fig. 6, there is a large parameter space. The estimates carried out here are representative only, but provide a guide from which to determine approximate errors in most such analyses. To restrict the parameter space, two assumptions were made:

1. The width of the patch, $\pm n \sigma_{\mathrm{s}}$, was taken as the width over which flux in the signal was summed.

2. "One percent" baselines were adopted; for each member of the family of baselines in Fig. 5, just enough width was allowed in the filter to include sufficient components so that when the true baselines were reconstructed after filtering, the maximum difference from the true baseline was one percent. Experience suggests that this is realistic to pessimistic. The situation is shown in Fig. 6.

Measured and true equivalent widths were calculated for Gaussian signals centered on baselines in the family of Fig. 5. This may be done analytically because of the additive nature of Fourier transforms. For the patched/filtered scan, the transform of two truncated Gaussians (regions $A$ and $C$ ) separated by a rectangular region ( $B$, corresponding to the patch), can be multiplied by the filter function

$F_{\mathrm{m}}(\omega)=F($ filter $) \times(F(A)+F(B)+F(C))$

to obtain the signal error after reverse transformation, as

$\Delta$ (flux) $=\int_{-a}^{+a}\left(F_{\mathrm{t}}(x)-F_{\mathrm{m}}(x)\right) \mathrm{d} x$

where $F_{\mathrm{t}}$ is the true flux and the signal flux is measured out to $\pm a$ about its centroid.

In practice it was simplest to use the FT system set up to analyze the real data (Laing et al. 1994 and in preparation). Checking such calculations is simple because there are at least three cases where geometrical analysis gives close approximation. Consider the following two examples.

1. The signal is very narrow in comparison to the scalelength (FWHM) of the baseline distortion, so that the baseline appears quasi-flat to it. For the geometry described in Figs. 5 and 6, the true equivalent width is $W_{\mathrm{t}}=\sqrt{2 \pi} h \sigma_{\mathrm{s}}$.

In this case the error on the baseline due to filtering (inclusion of too few components) dominates. Suppose this error is $\Delta(b+1)$. Then the ratio of measured to true equivalent width is

$\frac{W_{\mathrm{m}}}{W_{\mathrm{t}}}=\frac{\sqrt{2 \pi} h+\Delta 2 n}{\sqrt{2 \pi} h(1-\Delta)}$

where the flux is summed over $\pm n \sigma_{\mathrm{s}}$ about the signal centre.

2. If the signal is broad in comparison with the scan distortion scale-length, then the filtering of the patch has minimal effect. When it has no effect then from algebra of two Gaussians, a narrower one sitting centered on the broader one, the signal will be measured as

$$
\begin{aligned}
F_{\mathrm{m}}= & h(b+1) \sigma_{\mathrm{s}} \sqrt{2 \pi} \int_{-n}^{+n} \mathrm{e}^{-x^{2} / 2} \mathrm{~d} x \\
& +b \sigma_{\mathrm{b}} \sqrt{2 \pi} \int_{-n}^{+n} \mathrm{e}^{-x^{2} / 2} \mathrm{~d} x \\
& -2 n \sigma_{\mathrm{s}} b \mathrm{e}^{-\frac{\left(n \sigma_{\mathrm{s}}\right)^{2}}{2 \sigma_{\mathrm{b}}^{2}}}
\end{aligned}
$$

for measurement over $\pm n \sigma_{\mathrm{s}}$. The last two terms represent the area in the top of the Gaussian distortion which is included by virtue of the patch at $\pm n \sigma_{\mathrm{s}}$.

The measured equivalent width becomes

$W_{\mathrm{m}}=F_{\mathrm{m}} /\left(1+b \cdot \mathrm{e}^{-\frac{\left(n \sigma_{\mathrm{s}}\right)^{2}}{2 \sigma_{\mathrm{b}}^{2}}}\right)$. 


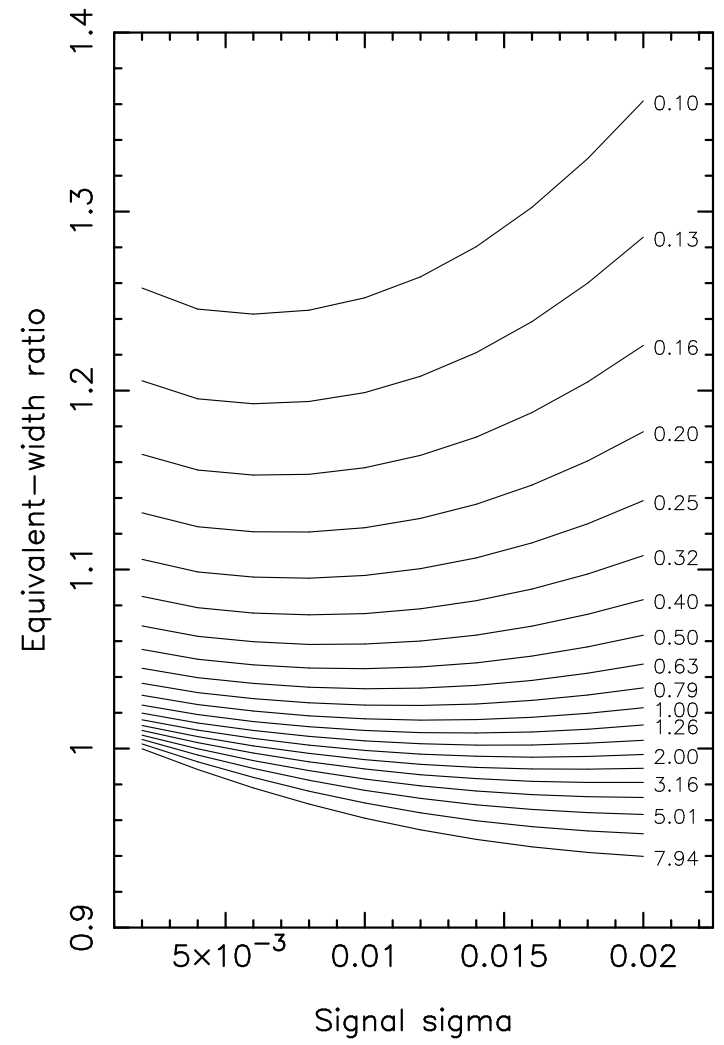

Fig. 7. Ratio of measured equivalent width to true equivalent width against $\sigma_{\mathrm{s}}$, the signal dispersion in units of scan length, for a Gaussian signal sitting at the peak of a continuum whose Gaussian FWHM $=0.628$ and $b=1.0$. The baseline has been estimated as described in the text, using a filter width to produce (in the absence of signal) a $1 \%$ deviation of the filtered baseline from the true baseline. The patch width and the width over which the flux is measured is $\pm 3 \sigma_{\mathrm{s}}$. The height of the signal Gaussian is $h(b+1)$, and the curves are computed for different signal strengths with values of $h$ as shown

The computed results (with which these approximations agree) are shown in Fig. 7 to Fig. 11. In Fig. 7, the error dependence on width of signal is shown for a given member of the continuum family in Fig. 5. For small and narrow signals, the error is almost totally due to the $1 \%$ baseline deviation. The error drops slightly as signal width increases, the total flux of the signal dominating the extra signal. But as signal width increases further, the error rises rapidly as the patching process includes some of the peak of the Gaussian scan distortion in the total flux estimation. For strong signals the situation is completely different; at small $\sigma_{\mathrm{s}}$ the estimate is accurate as the $1 \%$ baseline error is negligible. But as $\sigma_{\mathrm{s}}$ increases, the ratio drops below unity because the $\pm n \sigma_{\mathrm{s}}$ patch is not at the base of the signal; the excess signal now pulls up the (relatively weak) baseline, and this overestimate of the baseline dominates the error in measured equivalent width. Hence for very strong sources, as $\sigma_{\mathrm{s}}$ increases, the equivalent width becomes progressively underestimated. These effects are in general identifiable, depending on the signal-to-noise ratio (Fig. 6).

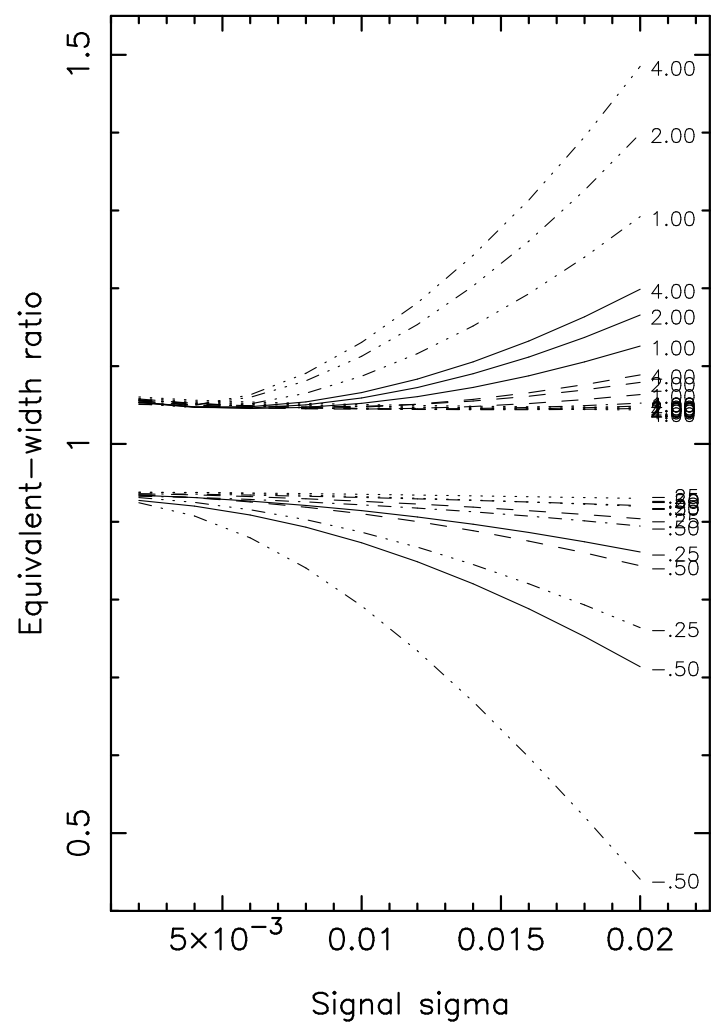

Fig. 8. Ratio of measured to true equivalent width versus width of signal in units of scan-length. The height parameter of the Gaussian signal (total height $=h(b+1)$ ) is fixed at $h=0.5$, and the patch width is fixed at $\pm 3 \sigma_{\mathrm{s}}$. The scale-length of the Gaussian distortion on the unit dc baseline is varied: FWHM = 0.25 scan-length, triple-dot - dash lines; FWHM $=0.395$, solid lines; FWHM $=0.628$, dashed lines; FWHM $=0.995$, dot-dash lines; and FWHM = 1.577 dotted lines. The amplitude of the distortion is varied, with $b$ taking five values, $-.25,-.5,1.0,2.0$ and 4.0, marked against the curves. There is a small asymmetry of $\sim 0.5 \%$ about the value of 1.0 , a second-order effect due to the patch pulling the filtered baseline towards it

In Fig. 8, a fixed value of $h$, signal height, was adopted, and the error is shown again as a function of signal width. Here the effects of the different distortions of baseline are shown, i.e. a representative set of the continuum family of Fig. 5 is introduced. The central gap is the effect of the $1 \%$ baseline fit; for a convex scan (positive $b$ ) it is to overestimate the equivalent width, while for a concave scan (negative $b$ ), it is to underestimate. For smaller scale-length 

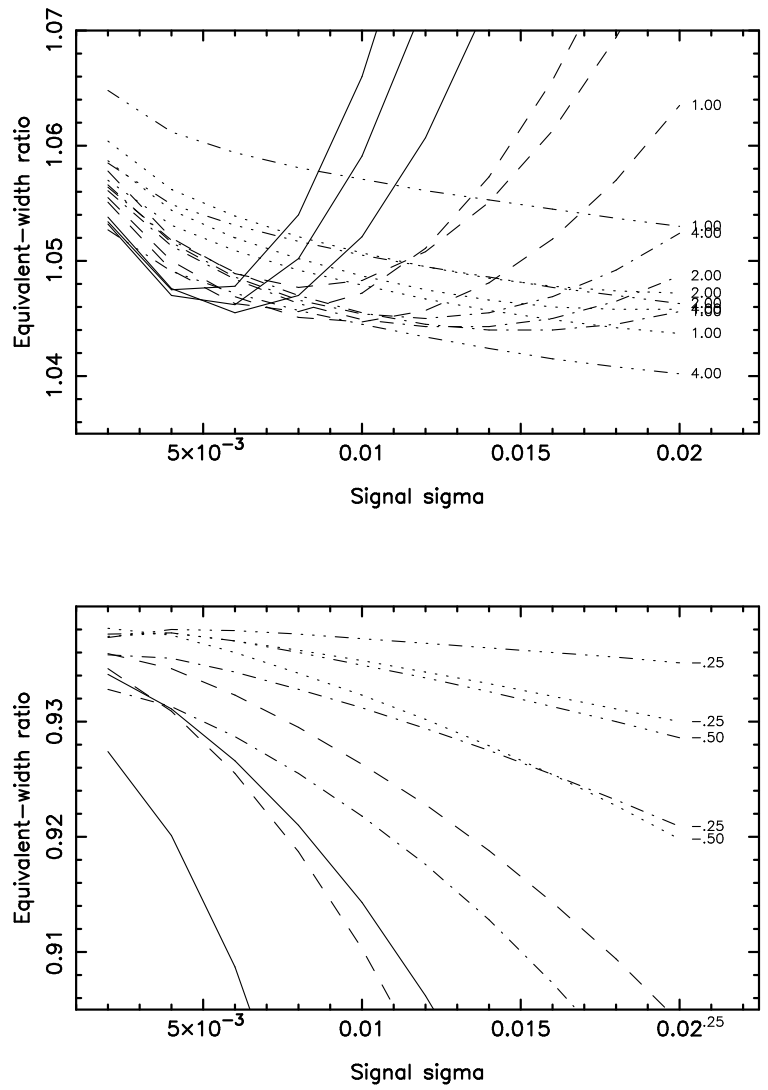

Fig. 9. As for Fig. 8, except that the vertical scale is much expanded to show what happens for the continua of lesser distortion. The curves are for Gaussian distortions on unit baselines of FWHM $=0.395$ scan length, solid lines; FWHM $=0.628$, dashed lines; FWHM $=0.995$, dot-dash lines; FWHM $=1.577$, dotted lines; and FWHM $=2.50$, triple-dot - dash lines

and for the larger $b$ values, the measured equivalent width deviates rapidly from the true value because of error in continuum estimate and (more important) the inclusion of baseline in the signal estimate. These effects diminish with decreasing $b$ and with increased FWHM. The error is drastically less for members of the family which curve gently, and Fig. 9 shows scales expanded about the central gap to illustrate the dependence of errors in these situations.

Figure 10 illustrates the effect of varying the patch width. For the weaker sources ( $h=0.1$ and 0.5$)$ the equivalent width is consistently overestimated for convex baselines. The overestimate increasing dramatically and monotonically with patch width because the patch lowers the effective continuum used to estimate the equivalent width while increasing the amount of continuum erroneously included in measurement of signal. For strong sources the ratio becomes insensitive to patch width as the continuum appears almost flat to the source. The dominant effect

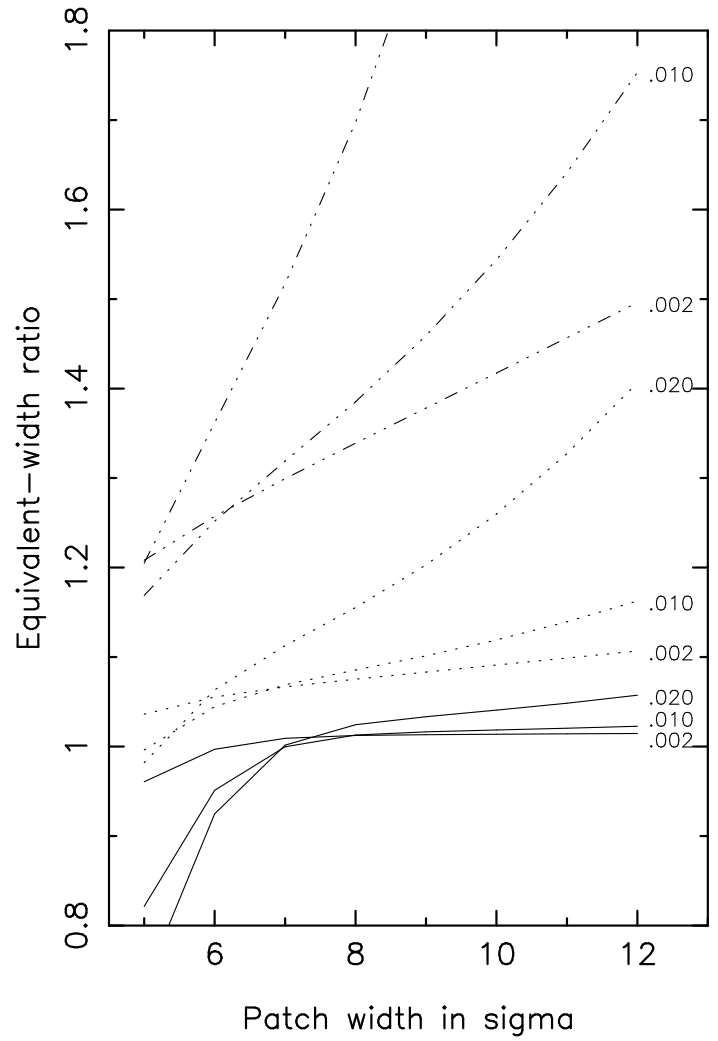

Fig. 10. Ratio of measured equivalent width to true equivalent width as a function of patch width. The Gaussian signal, of standard deviation $\sigma_{\mathrm{s}}=0.002,0.01$ or 0.02 as marked, is positioned at the crest of the distorted baseline with $b=1.0$ and FWHM $=0.628$. The dot-dash curves are for signal height $h=0.1$, the dotted curves for $h=0.5$, and the solid curves for $h=10.0$. The minimum components have been chosen to give a $1 \%$ maximum baseline error

is for short patches; as the width shrinks below $\pm 3.5 \sigma_{\mathrm{s}}$, signal is underestimated while the patch becomes placed relatively high into the signal, raising the estimated continuum significantly with respect to the true continuum. Both effects reduce the measured equivalent width.

The following points emerge from the error analysis.

- Except for very narrow signal, the baseline difference, i.e. the error in continuum assessment due to minimum-component fitting, will not dominate errors.

- The patch width is critical. For noisy situations in which the signal is relatively weak, it is imperative to choose a patch width which is as narrow as possible. Gaussians cut off quickly and for the weaker signals, patch and flux measurement should be confined to $\pm 3 \sigma_{\mathrm{s}}$, as determined from as accurate an estimate of $\sigma_{\mathrm{s}}$ as possible. For strong signals, the patch should be significantly broader. 


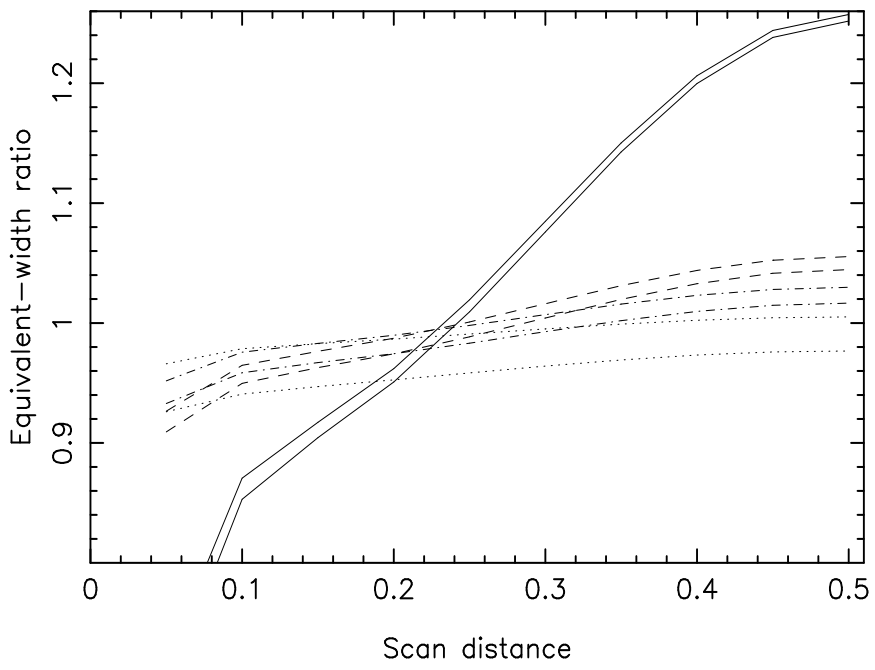

Fig. 11. Ratio of measured equivalent width to true equivalent width as a function of distance along scan. Previous error curves have all been computed with the signal centered at the $\max / \min$ of the baseline. Here the Gaussian signal is moved along the scan from the edge to the centre (at 0.5). The single continuum model adopted has a Gaussian of height $b=1.0$, $\mathrm{FWHM}=0.628$ sitting on a dc level of 1.0. The curves shown are for Gaussian signals of $h=0.1$ (solid lines), 0.5 (dashed lines), 1.0 (dash-dot lines), and 5.0 (dotted lines). Patch width and flux measurement is over $\pm 3 \sigma_{\mathrm{s}}$ in each case. The two lines for each signal height are for $\sigma_{\mathrm{s}}=0.002$ (upper) and 0.01 . The minimum components have been chosen to give a $1 \%$ maximum baseline error

- For even the weakest apparent signals it is crucial to patch over the region in which signal measurement is carried out. This may seem self-evident, but when signal is weak (e.g. Fig. 6e) it is tempting to fit a minimum-component continuum with minimal patching because the fit looks satisfactory. This is not so; the error introduced in the flux measurement may far exceed the noise uncertainty.

Two things must be borne in mind when using the present results to estimate errors on equivalent widths. Firstly, the worst-case situation has been examined in which the signal sits at the point of maximum inflection, at the centre of the continuum models. Figure 11 shows that for the Gaussian model continua on average the magnitude of the errors is about half these maximum values, the error on the $\sim$ linear flanks of the Gaussian being close to zero. Secondly, the curves have been computed on the basis of "1\%" continuum fits. These too are pessimistic; experience shows that the minimum-component baselines are generally more accurate than this.

The analysis indicates how rapidly errors in equivalent widths can escalate with non-linear continua, even when the procedures for continuum assessment and signal measurement are well defined. When yet broader wings are involved, the errors produced will be substantially greater.
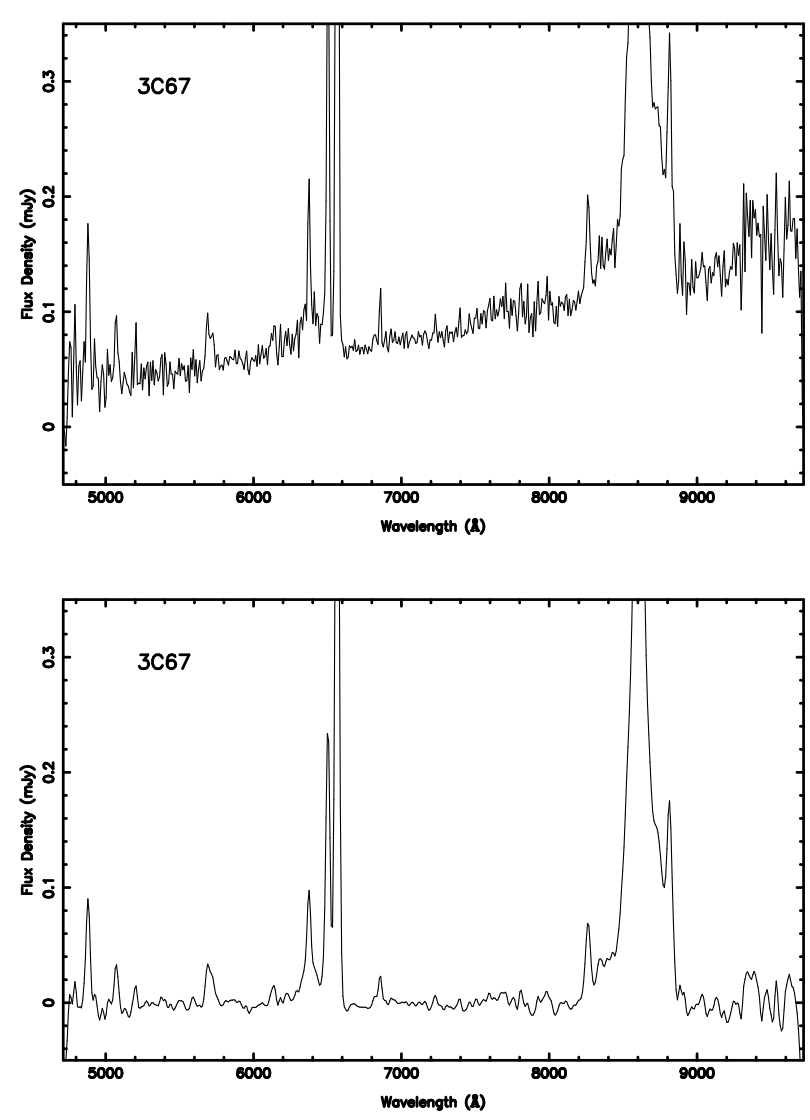

Fig. 12. The spectrum of 3 C 67 (Laing et al. 1994) to which bandpass filtering has been applied. The lower panel shows the results of removing low-frequency components, i.e. the continuum, by the process described above, and by removing the highest-frequency components to improve signal-to-noise ratio

The analysis goes some way to explaining why estimates of line-fluxes in the literature can differ by a factor of two, even with reasonable signal-to-noise.

\subsection{Further possibilities}

Harmonic analysis for scans (data-series) is powerful and versatile. Having assessed the harmonic content via an FT, formal techniques can be developed on the basis e.g. of known instrumental parameters to apply low and highfrequency filtering automatically to the data both to remove (or assess) the continuum and to improve signal-tonoise ratio. This is bandpass filtering, and an example is shown in Fig. 12. And indeed there are further uses of the methodology, Fig. 13 showing as an example the evaluation of the equivalent width of an absorption line in the midst of a strong emission line.

In addition the technique advocated here may be automated if some assumptions about the signal are made. For instance, if all signals are unresolved, an iterative procedure may be developed using differences between first 

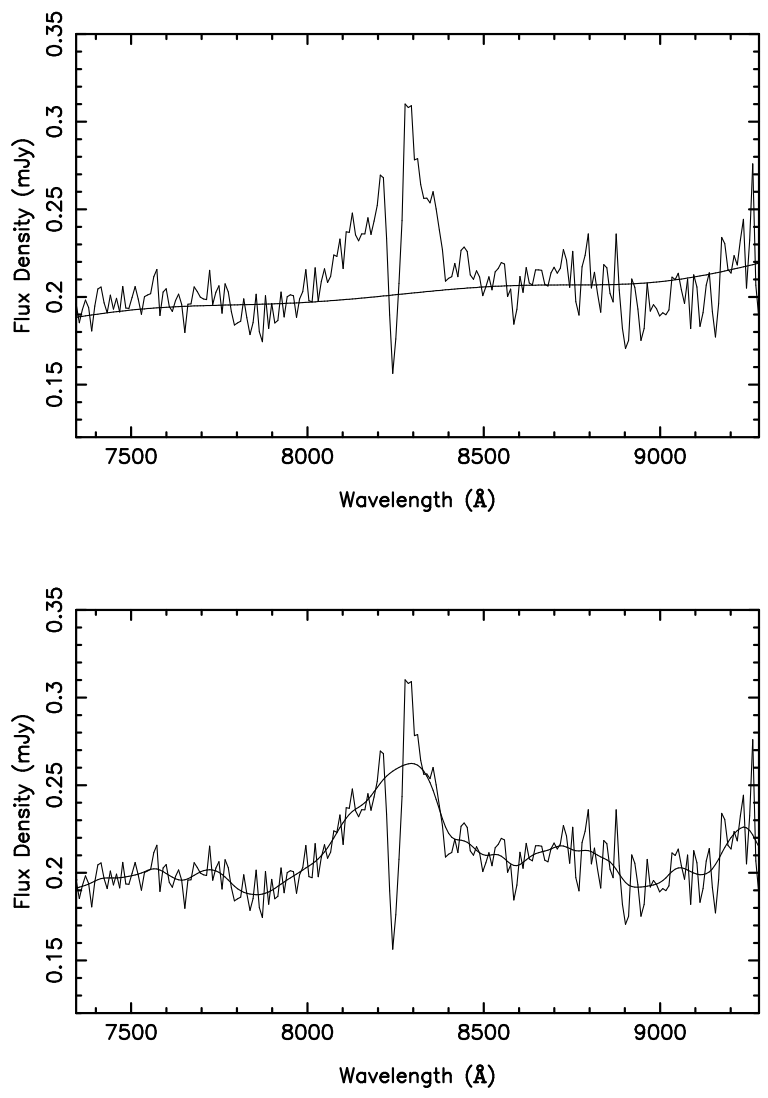

Fig. 13. A portion of the optical spectrum of $3 \mathrm{C} 191$ showing the broad emission line of MgII at $\sim 2800 \AA$ (rest frame), cut by a narrow absorption line. The upper panel shows the emission line together with a baseline determined as for 3C 47; the lower panel shows a "baseline" in which $\sigma=30$ (rather than $\sigma=3.0$ ) was used in the Gaussian taper, constructing a "baseline" from many more low-frequency components. From these two "continuum" estimates, parameters (equivalent width, etc.) of both the absorption and the emission line could be measured

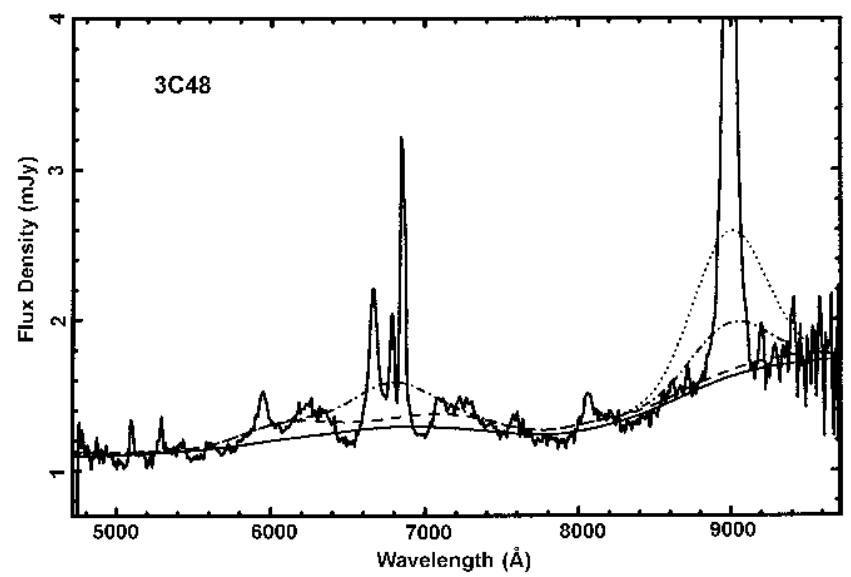

Fig. 14. The spectrum of $3 \mathrm{C} 48$, with baseline assessment by a fully objective procedure. The smooth lines show baseline iterations: 1 -dotted; 2 -dot-dash; 20 -dashed; 150 - full approximations to a baseline and the original data to decide upon regions to patch; patch widths are then simply the instrumental resolution width. A second iteration is then carried out with the new baseline to decide if additional regions need patching. For the more general case in which the signal is resolved, different algorithms may be appropriate. Figure 14 shows an example. As before, a "baseline array" is formed from which a baseline is constructed from the few lowest-spatial-frequency components. In the first instance, the baseline array is set as the data array, and the first iteration consists of forming a baseline from the lowest-spatial-frequency components. Each subsequent iteration consists of finding the largest difference between the previous baseline and the data array, and then replacing the data in the baseline array with the data from the previous baseline iteration, and \pm half-width of the instrument profile about this point. A different region of replacement was demanded for all subsequent iterations. The algorithm is very inefficient, but effective for virtually all the spectra tried so far, with the exception of spectra for which very strong broad emission/absorption lines occur at the ends of the scans. But almost all procedures struggle under this circumstance. Many different algorithms could be adopted to improve efficiency: broader patches, a line-list as a starting point, etc. There is resemblance in such procedures to the CLEAN technique (Högbom 1974) used in radio astronomy synthesis mapping.

Acknowledgements. I am grateful to Robert Laing, Charles Jenkins and Steve Unger for permission to use data before publication, and to Pierre Maxted for supplying me with the digitized version of the observation of RZ Cas. I appreciated helpful comments on drafts by David Carter, Charles Jenkins and a referee.

\section{References}

Bracewell R., 1965, The Fourier Transform and its Applications. McGraw-Hill, New York

Gaskill J.D., 1978, Linear Systems, Fourier Transforms and Optics. Wiley, New York

Högbom J.A., 1974, A\&AS 15, 417

Laing R.L., Jenkins C.R., Wall J.V., Unger S.W., 1994, in: Bicknell G.V., Dopita M.A., Quinn P.J. (eds.) The First Stromlo Symposium: The Physics of Active Galaxies, PASPC 54, 201

Martin M.A., 1959, Proc. IRE Trans Space Electronics and Telemetry SET-5, 33

Maxted P.F.L., Hill G., Hilditch R.W., 1994, A\&A 282, 821

Sargent W.L.W., Schechter P.L, Boksenberg A., Shortridge K., 1977, ApJ 212, 326

Shannon C.E., 1949, Proc. IRE 37, 10

Sivia D.S., Carlile C.J., 1992, J. Chem. Phys. 96, 170

Tonry J., Davis M., 1979, AJ 84, 1511

Wall J.V., Scheuer P.A.G., Pauliny-Toth I.I.K., Witzel A., 1982, MNRAS 198, 221

Whittaker E.T., 1915, Proc. Roy. Soc. Edinburgh A. 35, 181 